\title{
Gradient estimate of the heat kernel on modified graphs *
}

\author{
Satoshi Ishiwata ${ }^{\dagger}$ \\ Institute of Mathematics, University of Tsukuba, \\ Tsukuba-shi, Ibaraki, 305-8571, JAPAN
}

May 21, 2007

\begin{abstract}
We obtain a condition on the modification of graphs which guarantees the preservation of the Gaussian upper bound for the gradient of the heat kernel.
\end{abstract}

\section{Introduction}

Let us consider the simple random walk on an infinite graph $X$ and denote the kernel of the associated heat semigroup by $k_{n}$. Our interest is to see whether the gradient of the heat kernel $\nabla k_{n}$ has the Gaussian upper bound:

$$
\nabla k_{n}(x, y) \leq \frac{C}{\sqrt{n} V(x, \sqrt{n})} e^{-c d^{2}(x, y) / n} \quad \forall x, y \in X, \forall n \in \mathbb{N}^{*},
$$

where $V(x, r)$ is the volume of the ball centered at $x$ with radius $r$ for the combinatorial distance $d$ and $C, c$ are some positive constants.

In [7], Hebisch and Saloff-Coste proved that (1) is satisfied on discrete groups of polynomial volume growth. After that, the author generalized their result to the case of nilpotent covering graphs in [8] (see also [5], [6] and [9]). In the proof of all of them, the periodicity of the graph is used essentially. In this article, we prove that (1) is preserved under certain modification of the graph.

It should be noted that there is a connection between the estimate of (1) and the $L^{p}$-boundedness of the Riesz transform $\nabla \Delta^{-1 / 2}$ on non-compact Riemannian manifolds, where $\Delta$ is the Laplace-Beltrami operator. Let $M$ be a non-compact complete Riemannian manifold with volume doubling property

${ }^{*}$ Key words: gradient estimate of heat kernel, Gaussian bound, random walk on graph, modification of graph.

†ishiwata@math.tsukuba.ac.jp 
and $p_{t}$ the kernel of $e^{-t \Delta}$. We assume that $p_{t}$ satisfies the on-diagonal upper estimate

$$
p_{t}(x, x) \leq \frac{C}{V(x, \sqrt{t})},
$$

for all $x \in M, t>0$ and some constant $C>0$. By Theorem 1.4 in [1], the estimate

$$
\left|\nabla p_{t}(x, y)\right| \leq \frac{C}{\sqrt{t} V(x, \sqrt{t})} e^{-c d(x, y)^{2} / t}
$$

is sufficient to ensure the $L^{p}$-boundedness of the Riesz transform for all $1<$ $p<\infty$, namely there exists $C_{p}$ such that for any compact supported smooth function $f \in C_{0}^{\infty}(M)$,

$$
\left\|\nabla \Delta^{-1 / 2} f\right\|_{p} \leq C_{p}\|f\|_{p}, \quad 1<p<\infty .
$$

This fact is true also for graphs by Russ ([14]).

There are a lot of results about boundedness of the Riesz transform. See [1] and the literature therein.

\section{$1.1 \quad$ Notation and result}

First we fix a graph $X_{B}$ which is the basis of our modification. Let $X_{B}=$ $\left(V_{B}, E^{B}\right)$ be an oriented, locally finite connected infinite graph. Here $V_{B}$ is the set of vertices and $E^{B}$ is the set of oriented edges. For $e \in E^{B}$, we denote by $o(e), t(e)$ and $\bar{e}$ the origin of $e$, the end of $e$ and the inverse of $e$, respectively. We always assume that the set of edges of a graph includes their inverse edges. For $x \in V_{B}$, let $E_{x}^{B}=\left\{e \in E^{B} \mid o(e)=x\right\}$ and $m_{B}(x):=\operatorname{deg}_{B} x:=\# E_{x}^{B}$ the weight on $x$. For $x, y \in V_{B}, n \in \mathbb{N}$, the transition probability $p_{n}^{B}(x, y)$ for the simple random walk on $X_{B}$ is given by

$$
\begin{aligned}
& p_{0}^{B}(x, y)=\chi_{y}(x), \\
& p_{n}^{B}(x, y)=\sum_{\left(e_{1}, e_{2}, \ldots, e_{n}\right) \in C_{x, n}} p^{B}\left(e_{1}\right) p^{B}\left(e_{2}\right) \cdots p^{B}\left(e_{n}\right) \chi_{y}\left(t\left(e_{n}\right)\right) \quad n \in \mathbb{N}^{*},
\end{aligned}
$$

where $\chi_{y}$ is the characteristic function for $\{y\} \subset V_{B}, C_{x, n}$ is the set of paths from $x$ of length $n$ and $p^{B}(e)=\left(\operatorname{deg}_{B} o(e)\right)^{-1}$.

The transition operator $P_{B}$ associated to the simple random walk on $X_{B}$ is an operator acting on a function $f$ on $V_{B}$ by

$$
P_{B} f(x)=\sum_{e \in E_{x}^{B}} p^{B}(e) f(t(e)) .
$$

The $n$-th iteration $u(n, x)=P_{B}^{n} f(x)$ gives the solution of the heat equation

$$
\begin{aligned}
\left(\partial_{1}+\Delta\right) u(n, x) & =0 \\
u(0, x) & =f(x),
\end{aligned}
$$


where $\partial_{1} u(n, x)=u(n+1, x)-u(n, x)$ and

$$
\Delta u(n, x)=\sum_{e \in E_{x}^{B}} p^{B}(e)(u(n, o(e))-u(n, t(e))) .
$$

Then the kernel $k_{n}^{B}(x, y)$ of $P_{B}^{n}$ w.r.t. the measure $m_{B}$ is written as $k_{n}^{B}(x, y)=$ $p_{n}^{B}(x, y) m_{B}^{-1}(y)$.

For $x, y \in V_{B}$, let $d_{B}(x, y)$ be the combinatorial distance from $x$ to $y$ and $V_{B}(x, r):=\sum_{d_{B}(x, z) \leq r} m_{B}(z)$ the measure of $B_{B}(x, r)=\left\{y \in V_{B} \mid d_{B}(x, y) \leq\right.$ $r\}$. We assume that $X_{B}$ satisfies the volume doubling property, namely there exists a constant $C>0$ such that for any $x \in V_{B}$ and $r>0$,

$$
V_{B}(x, 2 r) \leq C V_{B}(x, r)
$$

It follows that $m_{B}$ is uniformly finite. Because, for any $y \sim x$, the neighbors in $V_{B}$,

$$
m_{B}(x) \leq \sum_{d_{B}(y, z) \leq 1} m_{B}(z)=V_{B}(y, 1) \leq C V_{B}(y, 1 / 2)=C m_{B}(y)
$$

Then we have

$C m_{B}(x)=C V_{B}(x, 1 / 2) \geq V_{B}(x, 1)=\sum_{d_{B}(x, y) \leq 1} m_{B}(y) \geq \sum_{d_{B}(x, y) \leq 1} \frac{1}{C} m_{B}(x)=\frac{1}{C} m_{B}^{2}(x)$,

namely

$$
m_{B}(x) \leq C^{2} \quad \forall x \in V_{B}
$$

We also assume that there exist positive constants $C$ and $c$ such that

$$
\begin{gathered}
k_{n}^{B}(x, y) \leq \frac{C}{V_{B}(x, \sqrt{n})} e^{-c d_{B}(x, y)^{2} / n}, \\
\nabla^{B} k_{n}^{B}(x, y) \leq \frac{C}{\sqrt{n} V_{B}(x, \sqrt{n})} e^{-c d_{B}(x, y)^{2} / n}
\end{gathered}
$$

for all $x, y \in V_{B}$ and $n \in \mathbb{N}^{*}$, where $\nabla^{B} k_{n}^{B}(x, y)$ is the gradient of $k_{n}^{B}$ for the first variable which is defined by

$$
\nabla^{B} k_{n}^{B}(x, y)=\left(\sum_{d_{B}(x, \omega) \leq 1}\left|k_{n}^{B}(\omega, y)-k_{n}^{B}(x, y)\right|^{2} p_{1}^{B}(x, \omega)\right)^{1 / 2} .
$$

In this paper, we consider the stability of (3) under the modification of $X_{B}=\left(V_{B}, E^{B}\right)$ given by

$$
X=(V, E)=\left(V_{B} \backslash V_{B}^{\prime},\left(E^{B} \cup \mathbb{A}^{+}\right) \backslash \mathbb{A}^{-}\right) .
$$


Here $\mathbb{A}^{+}$is the set of edges of a graph whose set of endpoints $A_{+}$is a subset in $V_{B}$ and $\mathbb{A}^{-}$is the set of edges of a subgraph in $X_{B}$ with the following conditions. If $\left(V_{B},\left(E^{B} \cup \mathbb{A}^{+}\right) \backslash \mathbb{A}^{-}\right)$is connected, this is the modified graph $X$. Then $V_{B}^{\prime}=\phi$. Otherwise, we choose a connected component as the modified graph $X$. In this case, we retake $\mathbb{A}^{-}$by adding all edges of other components and denote by $V_{B}^{\prime}$ the set of vertices of them. Then the chosen connected component can be written as $\left(V_{B} \backslash V_{B}^{\prime},\left(E^{B} \cup \mathbb{A}^{+}\right) \backslash \mathbb{A}^{-}\right)$.

We identify $\mathbb{A}^{+}$and $\mathbb{A}^{-}$with their associated graphs, respectively.

In other words, $X$ is constructed by the following three operations from $X_{B}$ :

1. Add edges $\mathbb{A}^{+}$.

2. Remove (retaken) edges $\mathbb{A}^{-}$.

3. Remove isolated vertices $V_{B}^{\prime}$.

For $x, y \in V$, let $d(x, y)$ be the combinatorial distance between $x$ and $y$ on $X$.

We assume that $X$ is uniformly finite, namely the weight $m(x)=\operatorname{deg} x$ on $x \in V$ is uniformly finite. Moreover, we assume that the natural inclusion $I:(X, d) \rightarrow\left(X_{B}, d_{B}\right)$ is a quasi-isometry, namely

(i) for a sufficiently large $\epsilon>0$, the $\epsilon$-neighborhood of $I(V)$ coincides with $V_{B}$

(ii) there exist constants $Q_{1} \geq 1$ and $Q_{2} \geq 0$ such that

$$
Q_{1}^{-1} d(x, y)-Q_{2} \leq d_{B}(I(x), I(y)) \leq Q_{1} d(x, y)+Q_{2}
$$

for all $x, y \in V$

(see [11]).

For $x, y \in V$, let $p_{n}(x, y)$ be the transition probability for the simple random walk on $X$ and $P^{n}$ the associated semigroup acting on the function on $V$. The kernel $k_{n}(x, y)$ of $P_{n}$ w.r.t. the weight $m(y)=\operatorname{deg} y$ is written as $k_{n}(x, y)=$ $p_{n}(x, y) m^{-1}(y)$. The gradient $\nabla k_{n}(x, y)$ for the first variable is also defined by the same manner of $\nabla^{B} k^{B}$ in (4). It is known that a quasi-isometric modification preserves the Gaussian upper bound for the heat kernel (2) (see [3]). Hence there exist positive constants $C$ and $c$ such that

$$
k_{n}(x, y) \leq \frac{C}{V(x, \sqrt{n})} e^{-c d(x, y)^{2} / n}
$$

for $x, y \in V$ and $n \in \mathbb{N}^{*}$, where $V(x, r):=\sum_{d(x, z) \leq r} m(z)$. We remark that $X$ also has the volume doubling property.

Let $A_{-}=o\left(\mathbb{A}^{-}\right) \cap V \subset V \subset V_{B}$ be the set of endpoints of $\mathbb{A}^{-}$in $V$. For $x \in V$, we denote by $V_{A}(x, r)$ the volume of $A=A_{+} \cup A_{-}$in $X$ defined by

$$
V_{A}(x, r)=\sum_{\substack{d\left(x, a_{+}\right) \leq r, a_{+} \in A_{+}}} m\left(a_{+}\right)+\sum_{\substack{d\left(x, a_{-}\right) \leq r, a_{-} \in A_{-}}} m\left(a_{-}\right) .
$$

Then the main result of this paper is the following: 
Theorem 1 If there exists a positive constant $M$ such that

$$
\frac{i V_{A}(x, \sqrt{i})}{V(x, \sqrt{i})}<M, \quad \sum_{\ell=1}^{\infty} \frac{V_{A}(x, \sqrt{\ell})}{V(x, \sqrt{\ell})}<M
$$

for all $x \in V$ and $i \in \mathbb{N}^{*}$, there exist positive constants $C$ and $c$ such that

$$
\nabla k_{n}(x, y) \leq \frac{C}{\sqrt{n} V(x, \sqrt{n})} e^{-c d(x, y)^{2} / n}
$$

for $x, y \in V$ and $n \in \mathbb{N}^{*}$.

In this paper, we do not allow the modification of adding vertices. This is because we need the injection of $X$ into $X_{B}$ to obtain the expression of $p_{n}(x, y)$ with $p_{n}^{B}(x, y)$ as in Lemma 2.1. However, our hypothesis of the modification and the assumption (7) of the Theorem may be strong in some cases. Indeed, there is a result that finite modifications of $\mathbb{Z}^{D}$ lattice graph preserve (8) in [4]. Moreover, as in Example 1.1, our result does not show the preservation of (8) under our modifications of $\mathbb{Z}^{D}$ if $D \leq 2$.

Example 1.1 Let $X_{B}=\left(V_{B}, E^{B}\right)$ be the D-dimensional square lattice graph, namely

$$
V_{B}=\mathbb{Z}^{D}, \quad E^{B}=\left\{(x, y) \in \mathbb{Z}^{D} \times \mathbb{Z}^{D}|| x-y \mid \leq 1\right\},
$$

where $|\cdot|$ is the Euclidean norm. For $\alpha<D$, let us put

$$
\mathbb{A}^{-}=E_{\mathbb{Z}^{\alpha}}=\cup_{x \in \mathbb{Z}^{\alpha}} E_{x}, \quad V_{B}^{\prime}=\mathbb{Z}^{\alpha},
$$

where

$$
\mathbb{Z}^{\alpha}=\left\{\left(x_{1}, x_{2}, \ldots, x_{\alpha}, 0, \ldots, 0\right) \in \mathbb{Z}^{D} \mid x_{i} \in \mathbb{Z}, 1 \leq i \leq \alpha\right\} .
$$

It is easy to see that if $\alpha \leq D-2$, the modified graph

$$
X=(V, E)=\left(V_{B} \backslash V_{B}^{\prime}, E^{B} \backslash \mathbb{A}^{-}\right)
$$

is connected. Since $B_{B}\left(V_{B} \backslash V_{B}^{\prime}, 1\right)=X_{B}$, the condition (i) of the quasi-isometry is satisfied. Since there are no added edges in $X, d_{B}(x, y) \leq d(x, y)$ for any $x, y \in V$. For $x, y \in V$, let $c=\left(x, c_{1}, c_{2}, \ldots, c_{k-1}, y\right)$ be a geodesic from $x$ to $y$ in $X_{B}$. If $c \cap \mathbb{Z}^{\alpha}=\phi, d(x, y)=d_{B}(x, y)$. If $c \cap \mathbb{Z}^{\alpha} \neq \phi$, it has one connected component. Then let us consider the parallel transformation $c \cap \mathbb{Z}^{\alpha}+e_{D} \subset$ $V_{B} \backslash V_{B}^{\prime}$ of $c \cap \mathbb{Z}^{\alpha}$, where $e_{D}=(0,0, \ldots, 0,1)$. Let $\left\{c_{i_{1}}, c_{i_{2}}\right\}=\partial\left(c \cap \mathbb{Z}^{\alpha}\right)\left(i_{1}<i_{2}\right)$, the boundary of $c \cap \mathbb{Z}^{\alpha}$. Since

$$
\begin{aligned}
& d\left(c_{i_{1}-1}, c_{i_{1}}+e_{D}\right) \leq 2, \quad d\left(c_{i_{2}}+e_{D}, c_{i_{2}+1}\right) \leq 2, \\
d(x, y) \leq & d\left(x, c_{i_{1}-1}\right)+d\left(c_{i_{1}-1}, c_{i_{1}}+e_{D}\right)+d\left(c_{i_{1}}+e_{D}, c_{i_{2}}+e_{D}\right) \\
& +d\left(c_{i_{2}}+e_{D}, c_{i_{2}+1}\right)+d\left(c_{i_{2}+1}, y\right) \\
\leq & d_{B}\left(x, c_{i_{1}-1}\right)+2+d_{B}\left(c_{i_{1}}, c_{i_{2}}\right)+2+d_{B}\left(c_{i_{2}+1}, y\right) \\
\leq & d_{B}(x, y)+2 .
\end{aligned}
$$


Then we have

$$
d(x, y)-2 \leq d_{B}(x, y) \leq d(x, y) .
$$

Hence $X$ is quasi-isometric to $X_{B}$ if $\alpha \leq D-2$.

It is proved that $\nabla^{B} k_{n}^{B}$ has the Gaussian estimate in [7]. The above theorem asserts that if $\alpha<D-2$, then the Gaussian estimate (8) for $\nabla k_{n}$ holds also on $X$.

Example 1.2 More generally, let us denote $X_{B}=\left(V_{B}, E^{B}\right)$ the Cayley graph of a finitely generated torsion free nilpotent group $\Gamma$ with a symmetric set $S$ of generators including the identity e of $\Gamma$. Namely, $X_{B}$ is the oriented graph defined by

$$
V_{B}=\Gamma, \quad(x, y) \in E^{B} \text { if } y^{-1} x \in S .
$$

From [7], the Gaussian upper bounds (2) and (3) hold on $X_{B}$.

As a modification of $X_{B}$, let us take a subgroup $H$ of $\Gamma$, namely denote by $\mathbb{A}^{-}=E_{H}=\cup_{h \in H} E_{h}$ and $V_{B}^{\prime}=H$. Let $D$ be the volume growth of $\Gamma$, namely $D$ is the number such that the volume of the ball $V_{B}(x, r)$ of centered at $x \in V_{B}$ with radius $r$ in $X_{B}$ satisfies

$$
C^{-1} r^{D} \leq V_{B}(x, r) \leq C r^{D}, \quad \forall x \in \Gamma, r \in \mathbb{N}^{*} .
$$

Similarly, let $\alpha$ be the number such that $B(x, r) \cap H$ satisfies

$$
V_{A}(x, r)=\sum_{z \in B(x, r) \cap H} m(z) \leq C r^{\alpha}, \quad \forall x \in V_{B}, r \in \mathbb{N}^{*} .
$$

In [10], it is proved that, if $\alpha \leq D-2$, the modified graph

$$
X=\left(V_{B} \backslash V_{B}^{\prime}, E^{B} \backslash \mathbb{A}^{-}\right)
$$

is quasi-isometric to $X_{B}$. The previous theorem asserts that if $\alpha<D-2$, then the Gaussian estimate (8) for $\nabla k_{n}$ holds also on $X$.

\section{Proof of the Theorem}

\section{$2.1 \quad$ Difference between $p_{n}^{B}$ and $p_{n}$}

For $a_{+} \in A_{+} \subset V$ and $a_{-} \in A_{-} \subset V$, let

$$
\begin{aligned}
& \operatorname{deg}_{+} a_{+}:=\# \mathbb{A}_{a_{+}}^{+}:=\#\left\{e_{+} \in \mathbb{A}^{+} \mid o\left(e_{+}\right)=a_{+}\right\} \\
& \operatorname{deg}_{-} a_{-}:=\# \mathbb{A}_{a_{-}}^{-}:=\#\left\{e_{-} \in \mathbb{A}^{-} \mid o\left(e_{-}\right)=a_{-}\right\}
\end{aligned}
$$

Let us denote $P_{+}$and $P_{-}$the transition operator on $\mathbb{A}^{+}$and $\mathbb{A}^{-}$, respectively. Then we have the following: 
Lemma 2.1 For $x, y \in V$ and $n \geq 1$,

$$
\begin{aligned}
p_{n}(x, y)=p_{n}^{B}(x, y) & -\sum_{\ell=1}^{n} \sum_{a_{+} \in A_{+}} p_{n-\ell}\left(x, a_{+}\right) \frac{\operatorname{deg}_{+} a_{+}}{\operatorname{deg} a_{+}}\left(p_{\ell}^{B}\left(a_{+}, y\right)-P_{+} p_{\ell-1}^{B}\left(a_{+}, y\right)\right) \\
& +\sum_{\ell=1}^{n} \sum_{a_{-} \in A_{-}} p_{n-\ell}\left(x, a_{-}\right) \frac{\operatorname{deg}_{-} a_{-}}{\operatorname{deg} a_{-}}\left(p_{\ell}^{B}\left(a_{-}, y\right)-P_{-} p_{\ell-1}^{B}\left(a_{-}, y\right)\right) .
\end{aligned}
$$

Proof. For a real valued function $f$ on $V_{B}$, let $I^{*} f$ be the pull-back to the function on $V$ by the inclusion $I: V \rightarrow V_{B}$. First, we show the following:

$$
\begin{aligned}
P\left(I^{*} f\right)(x)=I^{*}\left(P_{B} f\right)(x) & -\sum_{a_{+} \in A_{+}} \chi_{a_{+}}(x) \frac{\operatorname{deg}_{+} a_{+}}{\operatorname{deg} a_{+}}\left(I^{*}\left(P_{B} f\right)\left(a_{+}\right)-P_{+}\left(\left.f\right|_{\mathbb{A}^{+}}\right)\left(a_{+}\right)\right) \\
& +\sum_{a_{-} \in A_{-}} \chi_{a_{-}}(x) \frac{\operatorname{deg} a_{-}}{\operatorname{deg} a_{-}}\left(I^{*}\left(P_{B} f\right)\left(a_{-}\right)-P_{-}\left(\left.f\right|_{\mathbb{A}^{-}}\right)\left(a_{-}\right)\right) .
\end{aligned}
$$

When $x \notin A_{+} \cup A_{-}$, clearly $P\left(I^{*} f\right)(x)=I^{*}\left(P_{B} f\right)(x)$. In the case of $x=a_{+} \in$ $A_{+} \backslash A_{-}$,

$$
\begin{aligned}
P\left(I^{*} f\right)\left(a_{+}\right) & =\frac{1}{\operatorname{deg} a_{+}} \sum_{e \in E_{a_{+}}} I^{*} f(t(e))=\frac{1}{\operatorname{deg} a_{+}} \sum_{e \in E_{a_{+}}} f(I(t(e))) \\
& =\frac{1}{\operatorname{deg} a_{+}}\left(\sum_{e_{B} \in E_{I\left(a_{+}\right)}^{B}} f\left(t\left(e_{B}\right)\right)+\sum_{e_{+} \in \mathbb{A}_{I\left(a_{+}\right)}^{+}} f\left(t\left(e_{+}\right)\right)\right) \\
& =\frac{\operatorname{deg} B a_{+}}{\operatorname{deg} a_{+}} \cdot \frac{1}{\operatorname{deg}_{B} a_{+}} \sum_{e_{B} \in E_{I\left(a_{+}\right)}^{B}} f\left(t\left(e_{B}\right)\right)+\frac{1}{\operatorname{deg} a_{+}} \sum_{e_{+} \in \mathbb{A}_{I\left(a_{+}\right)}^{+}} f\left(t\left(e_{+}\right)\right) \\
& =\frac{\operatorname{deg} a_{+}-\operatorname{deg}_{+} a_{+}}{\operatorname{deg} a_{+}} P_{B} f\left(I\left(a_{+}\right)\right)+\frac{1}{\operatorname{deg} a_{+}} \sum_{e_{+} \in \mathbb{A}_{I\left(a_{+}\right)}^{+}} f\left(t\left(e_{+}\right)\right) \\
& =P_{B} f\left(I\left(a_{+}\right)\right)-\frac{\operatorname{deg}_{+} a_{+}}{\operatorname{deg} a_{+}}\left(P_{B} f\left(I\left(a_{+}\right)\right)-\frac{1}{\operatorname{deg}_{+} a_{+}} \sum_{e_{+} \in \mathbb{A}_{I\left(a_{+}\right)}^{+}} f\left(t\left(e_{+}\right)\right)\right) \\
& =I^{*}\left(P_{B} f\right)\left(a_{+}\right)-\frac{\operatorname{deg} a_{+} a_{+}}{\operatorname{deg} a_{+}}\left(I^{*}\left(P_{B} f\right)\left(a_{+}\right)-I^{*} P_{+} f\left(a_{+}\right)\right) .
\end{aligned}
$$


When $x=a_{-} \in A_{-} \backslash A_{+}$, similarly we have

$$
\begin{aligned}
P\left(I^{*} f\right)\left(a_{-}\right) & =\frac{1}{\operatorname{deg} a_{-}}\left(\sum_{e_{B} \in E_{I\left(a_{-}\right)}^{B}} f\left(t\left(e_{B}\right)\right)-\sum_{e_{-} \in \mathbb{A}_{I\left(a_{-}\right)}^{-}} f\left(t\left(e_{-}\right)\right)\right) \\
& =\frac{\operatorname{deg} a_{-}+\operatorname{deg} a_{-}}{\operatorname{deg} a_{-}} P_{B} f\left(I\left(a_{-}\right)\right)-\frac{1}{\operatorname{deg} a_{-}} \sum_{e_{-} \in \mathbb{A}_{I\left(a_{-}\right)}^{-}} f\left(t\left(e_{-}\right)\right) \\
& =I^{*}\left(P_{B} f\right)\left(a_{-}\right)+\frac{\operatorname{deg} a_{-}}{\operatorname{deg} a_{-}}\left(I^{*}\left(P_{B} f\right)\left(a_{-}\right)-I^{*} P_{-} f\left(a_{-}\right)\right) .
\end{aligned}
$$

Finally, if $x=a \in A_{+} \cap A_{-}$, we have

$$
\begin{aligned}
P\left(I^{*} f\right)(a)= & \frac{1}{\operatorname{deg} a}\left(\sum_{e_{B} \in E_{I(a)}^{B}} f\left(I\left(t\left(e_{B}\right)\right)\right)+\sum_{e_{+} \in \mathbb{A}_{I(a)}^{+}} f\left(t\left(e_{+}\right)\right)-\sum_{e_{-} \in \mathbb{A}_{I(a)}^{-}} f\left(t\left(e_{-}\right)\right)\right) \\
= & \frac{\operatorname{deg} a-\operatorname{deg}_{+} a+\operatorname{deg}_{-} a}{\operatorname{deg} a} P_{B} f(I(a))+\frac{1}{\operatorname{deg} a} \sum_{e_{+} \in \mathbb{A}_{I(a)}^{+}} f\left(t\left(e_{+}\right)\right) \\
& -\frac{1}{\operatorname{deg} a} \sum_{e_{-} \in \mathbb{A}_{I(a)}^{-}} f\left(t\left(e_{-}\right)\right) \\
= & I^{*}\left(P_{B} f\right)(a)-\frac{\operatorname{deg}_{+} a}{\operatorname{deg} a}\left(I^{*}\left(P_{B} f\right)(a)-I^{*} P_{+} f(a)\right) \\
& +\frac{\operatorname{deg}_{-} a}{\operatorname{deg} a}\left(I^{*}\left(P_{B} f\right)(a)-I^{*} P_{-} f(a)\right) .
\end{aligned}
$$

Hence (10) is obtained. 
From the definition of $p_{n}(x, y)$, we have

$$
\begin{aligned}
p_{n}(x, y)= & \sum_{\left(e_{1}, e_{2}, \ldots, e_{n-1}\right) \in C_{x, n-1}} p\left(e_{1}\right) p\left(e_{2}\right) \cdots p\left(e_{n-1}\right) \sum_{e \in E_{t}\left(e_{n-1}\right)} p(e) p_{0}^{B}\left(t\left(e_{n-1}\right), y\right) \\
= & \sum_{\left(e_{1}, e_{2}, \ldots e_{n-1}\right) \in C_{x, n-1}} p\left(e_{1}\right) p\left(e_{2}\right) \cdots p\left(e_{n-1}\right) \\
& \times\left(P_{B} p_{0}^{B}\left(t\left(e_{n-1}\right), y\right)-\sum_{a_{+} \in A_{+}} \chi_{a_{+}}\left(t\left(e_{n-1}\right)\right) \frac{\operatorname{deg}_{+} a_{+}}{\operatorname{deg} a_{+}}\left(P_{B} p_{0}^{B}\left(a_{+}, y\right)-P_{+} p_{0}^{B}\left(a_{+}, y\right)\right)\right. \\
& \left.+\sum_{a_{-} \in A_{-}} \chi_{a_{-}}\left(t\left(e_{n-1}\right)\right) \frac{\operatorname{deg} a_{-}}{\operatorname{deg} a_{-}}\left(P_{B} p_{0}^{B}\left(a_{-}, y\right)-P_{-} p_{0}^{B}\left(a_{-}, y\right)\right)\right) \\
= & \sum_{\left(e_{1}, e_{2}, \ldots e_{n-1}\right) \in C_{x, n-1}} p\left(e_{1}\right) p\left(e_{2}\right) \cdots p\left(e_{n-1}\right) p_{1}^{B}\left(t\left(e_{n-1}\right), y\right) \\
& -\sum_{a_{+} \in A_{+}} p_{n-1}\left(x, a_{+}\right) \frac{\operatorname{deg} a_{+} a_{+}}{\operatorname{deg} a_{+}}\left(p_{1}^{B}\left(a_{+}, y\right)-P_{+} p_{0}^{B}\left(a_{+}, y\right)\right) \\
& +\sum_{a_{-} \in A_{-}} p_{n-1}\left(x, a_{-}\right) \frac{\operatorname{deg}_{-} a_{-}}{\operatorname{deg} a_{-}}\left(p_{1}^{B}\left(a_{-}, y\right)-P_{-} p_{0}^{B}\left(a_{-}, y\right)\right) .
\end{aligned}
$$

By using (10) inductively, we conclude

$$
\begin{aligned}
p_{n}(x, y)=p_{n}^{B}(x, y) & -\sum_{\ell=1}^{n} \sum_{a_{+} \in A_{+}} p_{n-\ell}\left(x, a_{+}\right) \frac{\operatorname{deg}_{+} a_{+}}{\operatorname{deg} a_{+}}\left(p_{\ell}^{B}\left(a_{+}, y\right)-P_{+} p_{\ell-1}^{B}\left(a_{+}, y\right)\right) \\
& +\sum_{\ell=1}^{n} \sum_{a_{-} \in A_{-}} p_{n-\ell}\left(x, a_{-}\right) \frac{\operatorname{deg}_{-} a_{-}}{\operatorname{deg} a_{-}}\left(p_{\ell}^{B}\left(a_{-}, y\right)-P_{-} p_{\ell-1}^{B}\left(a_{-}, y\right)\right) .
\end{aligned}
$$

\subsection{Estimate by induction}

We prove the Theorem by an induction on time. Since $\nabla k_{1}(x, y)$ is bounded w.r.t. $x, y$ and the support of $\nabla k_{1}(x, y)$ as a function of $y \in V$ is included in $B(x, 2)$, it is trivial to see that there exist positive constants $T_{1}$ and $t_{1}$ such that

$$
\nabla k_{1}(x, y) \leq \frac{T_{1}}{V(x, 1)} e^{-t_{1} d^{2}(x, y)} .
$$

Next, for $\nu>1$, let us assume that there exist positive constants $T_{\nu}$ and $t_{\nu}$ such that

$$
\nabla k_{i}(x, y) \leq \frac{T_{\nu}}{\sqrt{i} V(x, \sqrt{i})} e^{-t_{\nu} d^{2}(x, y) / i}
$$

for all $x, y \in V$ and $1 \leq i \leq \nu-1$. Let us separate $\nu=m+n$ so that $m=n$ or $m=n+1$ depending on whether $\nu$ is even or odd. By the Cauchy-Schwarz 
inequality, we have

$$
e^{2 t_{\nu} d^{2}(x, y) / \nu} \nabla k_{\nu}(x, y) \leq\left\|e^{4 t_{\nu} d^{2}(x, \cdot) / n} \nabla k_{n}(x, \cdot)\right\|_{L^{2}}\left\|e^{4 t_{\nu} d^{2}(\cdot, y) / m} k_{m}(\cdot, y)\right\|_{L^{2}} .
$$

Since $k_{m}$ has the Gaussian upper bound (6), if $t_{\nu}$ is sufficiently small,

$$
\left\|e^{4 t_{\nu} d^{2}(\cdot, y) / m} k_{m}(\cdot, y)\right\|_{L^{2}} \leq \frac{C^{\prime}\left(t_{\nu}\right)}{V^{1 / 2}(y, \sqrt{m})} \leq \frac{C\left(t_{\nu}\right)}{V^{1 / 2}(y, \sqrt{\nu})} .
$$

We aim to show that

$$
\left\|e^{4 t_{\nu} d^{2}(x, \cdot) / n} \nabla k_{n}(x, \cdot)\right\|_{L^{2}} \leq \frac{C\left(t_{\nu}\right)\left(T_{\nu}+c\left(t_{\nu}\right)\right)^{1 / 2}}{\sqrt{n} V^{1 / 2}(x, \sqrt{n})} .
$$

For $n=1$, this is trivial. Thus we always assume that $n>1$.

By using Lemma 2.1, we have

$$
\begin{aligned}
& \left\|e^{4 t_{\nu} d^{2}(x, \cdot) / n} \nabla k_{n}(x, \cdot)\right\|_{L^{2}}^{2}=\sum_{z \in V} e^{8 t_{\nu} d^{2}(x, z) / n} \sum_{d(x, \omega) \leq 1}\left|k_{n}(\omega, z)-k_{n}(x, z)\right|^{2} p_{1}(x, \omega) m(z) \\
& =\sum_{z \in V} e^{8 t_{\nu} d^{2}(x, z) / n} \sum_{d(x, \omega) \leq 1}\left|p_{n}(\omega, z)-p_{n}(x, z)\right|^{2} p_{1}(x, \omega) m(z)^{-1} \\
& =\sum_{z \in V} e^{8 t_{\nu} d^{2}(x, z) / n} \sum_{d(x, \omega) \leq 1} \mid p_{n}^{B}(\omega, z)-p_{n}^{B}(x, z) \\
& -\left.\sum_{\ell=1}^{n} \sum_{i=+,-} i \sum_{a_{i} \in A_{i}}\left(p_{n-\ell}\left(\omega, a_{i}\right)-p_{n-\ell}\left(x, a_{i}\right)\right) \frac{\operatorname{deg}_{i} a_{i}}{\operatorname{deg} a_{i}}\left(p_{\ell}^{B}\left(a_{i}, z\right)-P_{i} p_{\ell-1}^{B}\left(a_{i}, z\right)\right)\right|^{2} \\
& \times p_{1}(x, \omega) m(z)^{-1} \\
& \leq 2 \sum_{z \in V} e^{8 t_{\nu} d^{2}(x, z) / n} \sum_{d(x, \omega) \leq 1}\left|p_{n}^{B}(\omega, z)-p_{n}^{B}(x, z)\right|^{2} p_{1}(x, \omega) m(z)^{-1} \\
& +2 \sum_{z \in V} e^{8 t_{\nu} d^{2}(x, z) / n} \sum_{d(x, \omega) \leq 1} \mid \sum_{\ell=1}^{n} \sum_{i=+,-} i \sum_{a_{i} \in A_{i}}\left(p_{n-\ell}\left(\omega, a_{i}\right)-p_{n-\ell}\left(x, a_{i}\right)\right) \\
& \left.\frac{\operatorname{deg}_{i} a_{i}}{\operatorname{deg} a_{i}}\left(p_{\ell}^{B}\left(a_{i}, z\right)-P_{i} p_{\ell-1}^{B}\left(a_{i}, z\right)\right)\right|^{2} p_{1}(x, \omega) m(z)^{-1} \\
& =I_{1}(n, x)+I_{2}(n, x) .
\end{aligned}
$$

We estimate $I_{1}$ and $I_{2}$ separately.

\subsection{Estimate of $I_{1}$}

Let us recall the property of $X_{B}$ and $X$. Since $m_{B}$ and $m$ are uniformly finite and the inclusion $I: V \rightarrow V_{B}$ is a quasi-isometry between $X$ and $X_{B}$, there exists $C>0$ depending only on $M_{B}=\max _{x \in V_{B}} m_{B}(x), Q_{1}$ and $Q_{2}$ in (5) such that

$$
I_{1}(n, x) \leq C \sum_{z \in V_{B}} e^{8 t_{\nu} Q_{1}^{2} d_{B}^{2}(x, z) / n} \sum_{d_{B}(x, \omega) \leq Q_{1}+Q_{2}}\left|k_{n}^{B}(\omega, z)-k_{n}^{B}(x, z)\right|^{2} m_{B}(z) .
$$


For $d_{B}(x, \omega) \leq Q_{1}+Q_{2}$, it is easy to see that

$$
\left|k_{n}^{B}(\omega, z)-k_{n}^{B}(x, z)\right|^{2} \leq\left(Q_{1}+Q_{2}\right) M_{B}^{2} \sum_{d_{B}\left(x, \omega^{\prime}\right) \leq Q_{1}+Q_{2}}\left|\nabla^{B} k_{n}^{B}\left(\omega^{\prime}, z\right)\right|^{2} .
$$

Hence $I_{1}(n, x)$ is estimated by

$$
C \sum_{z \in V_{B}} e^{8 t_{\nu} Q_{1}^{2} d_{B}^{2}(x, z) / n} \sum_{d_{B}(x, \omega) \leq Q_{1}+Q_{2}}\left|\nabla^{B} k_{n}^{B}(\omega, z)\right|^{2} m_{B}(z) .
$$

Since $\nabla^{B} k_{n}^{B}$ has the Gaussian upper bound (3),

$$
\begin{aligned}
& \sum_{z \in V_{B}} e^{8 t_{\nu} Q_{1}^{2} d_{B}^{2}(x, z) / n} \sum_{d_{B}(x, \omega) \leq Q_{1}+Q_{2}}\left|\nabla^{B} k_{n}^{B}(\omega, z)\right|^{2} m_{B}(z) \\
& \leq C \sum_{z \in V_{B}} e^{8 t_{\nu} Q_{1}^{2} d_{B}^{2}(x, z) / n} \sum_{d_{B}(x, \omega) \leq Q_{1}+Q_{2}} \frac{C^{2}}{n V(\omega, \sqrt{n})^{2}} e^{-2 c d_{B}^{2}(\omega, z) / n} m_{B}(z) \\
& \leq \frac{C^{\prime}}{n V(x, \sqrt{n})^{2}} \sum_{z \in V_{B}} e^{-\left(2 c-8 t_{\nu} Q_{1}^{2}\right) d_{B}^{2}(x, z) / n} m_{B}(z) .
\end{aligned}
$$

Consequently, if $t_{\nu}<c / 4 Q_{1}^{2}$, there exists $C\left(t_{\nu}\right)$ independent of $T_{\nu}$ such that

$$
I_{1}(n, x) \leq \frac{C\left(t_{\nu}\right)}{n V(x, \sqrt{n})}
$$

which gives a desired bound (11).

\subsection{Estimate of $I_{2}$}

In order to make the induction work, we estimate $I_{2}$ by the following:

$$
\begin{aligned}
& 2 \sum_{z \in V} \sum_{i, j=+,-\ell_{1}, \ell_{2}=1} \sum_{a_{1 i} \in A_{i}} \sum_{a_{2 j} \in A_{j}} e^{8 t_{\nu} d^{2}(x, z) / n} \\
& \sum_{d(x, \omega) \leq 1}\left|k_{n-\ell_{1}}\left(\omega, a_{1 i}\right)+k_{n-\ell_{1}}\left(x, a_{1 i}\right)\right| \operatorname{deg}_{i} a_{1 i}\left|k_{\ell_{1}}^{B}\left(a_{1 i}, z\right)-P_{i} k_{\ell_{1}-1}^{B}\left(a_{1 i}, z\right)\right| \\
& \times\left|k_{n-\ell_{2}}\left(\omega, a_{2 j}\right)-k_{n-\ell_{2}}\left(x, a_{2 j}\right)\right| \operatorname{deg}_{j} a_{2 j}\left|k_{\ell_{2}}^{B}\left(a_{2 j}, z\right)-P_{j} k_{\ell_{2}-1}^{B}\left(a_{2 j}, z\right)\right| \\
& \times m_{B}(z)^{2} m(x)^{-1} m(z)^{-1} .
\end{aligned}
$$


By the Cauchy-Schwarz inequality for $\omega, I_{2}$ is less than

$$
\begin{aligned}
& 2 \sum_{z \in V} \sum_{i, j=+,-\ell_{1}, \ell_{2}=1} \sum_{a_{1 i} \in A_{i}} \sum_{a_{2 j} \in A_{j}} e^{8 t_{\nu} d^{2}(x, z) / n} \\
& \times\left(\sum_{\left.d_{(} x, \omega\right) \leq 1}\left|k_{n-\ell_{1}}\left(\omega, a_{1 i}\right)+k_{n-\ell_{1}}\left(x, a_{1 i}\right)\right|^{2} m(x)^{-1}\right)^{1 / 2} \operatorname{deg}_{i} a_{1 i} \\
& \times\left|k_{\ell_{1}}^{B}\left(a_{1 i}, z\right)-P_{i} k_{\ell_{1}-1}^{B}\left(a_{1 i}, z\right)\right|\left(\sum_{d(x, \omega) \leq 1}\left|k_{n-\ell_{2}}\left(\omega, a_{2 j}\right)-k_{n-\ell_{2}}\left(x, a_{2 j}\right)\right|^{2} p_{1}(x, \omega)\right)^{1 / 2} \\
& \times \operatorname{deg}_{j} a_{2 j}\left|k_{\ell_{2}}^{B}\left(a_{2 j}, z\right)-P_{j} k_{\ell_{2}-1}^{B}\left(a_{2 j}, z\right)\right| m_{B}(z)^{2} m(z)^{-1} \\
& \leq 2 \sum_{z \in V} e^{8 t_{\nu}^{2} d(x, z) / n} \sum_{\ell_{1}=1}^{n} \sum_{i=+,-} \sum_{a_{1 i} \in A_{i}} 2 \sup _{d(x, \omega) \leq 1} k_{n-\ell_{1}}\left(\omega, a_{1 i}\right) \operatorname{deg}_{i} a_{1 i} \\
& \times\left|k_{\ell_{1}}^{B}\left(a_{1 i}, z\right)-P_{i} k_{\ell_{1}-1}^{B}\left(a_{1 i}, z\right)\right| \sum_{\ell_{2}=1}^{n} \sum_{j=+,-} \sum_{a_{2 j} \in A_{j}} \nabla k_{n-\ell_{2}}\left(x, a_{2 j}\right) \operatorname{deg}_{j} a_{2 j} \\
& \times\left|k_{\ell_{2}}^{B}\left(a_{2 j}, z\right)-P_{j} k_{\ell_{2}-1}^{B}\left(a_{2 j}, z\right)\right| m_{B}(z)^{2} m(z)^{-1} \text {. }
\end{aligned}
$$

Using the same arguments in (12),

$$
\begin{aligned}
& \left|k_{\ell}^{B}\left(a_{i}, z\right)-P_{i} k_{\ell-1}^{B}\left(a_{i}, z\right)\right| \\
& =\left|\left(k_{\ell}^{B}\left(a_{i}, z\right)-k_{\ell-1}^{B}\left(a_{i}, z\right)\right)+\left(k_{\ell-1}^{B}\left(a_{i}, z\right)-P_{i} k_{\ell-1}^{B}\left(a_{i}, z\right)\right)\right| \\
& \leq \frac{1}{\operatorname{deg} a_{i}} \sum_{e_{B} \in E_{a_{i}}^{B}}\left|k_{\ell-1}^{B}\left(t\left(e_{B}\right), z\right)-k_{\ell-1}^{B}\left(a_{i}, z\right)\right|+\frac{1}{\operatorname{deg}_{i} a_{i}} \sum_{e_{i} \in \mathbb{B}_{a_{i}}^{i}}\left|k_{\ell-1}^{B}\left(a_{i}, z\right)-k_{\ell-1}^{B}\left(t\left(e_{i}\right), z\right)\right| \\
& \leq \nabla^{B} k_{\ell-1}^{B}\left(a_{i}, z\right)+M_{B} \# B_{B}\left(a_{i}, Q_{1}+Q_{2}\right) \sum_{d_{B}\left(a_{i}, \omega\right) \leq Q_{1}+Q_{2}} \nabla^{B} k_{\ell-1}^{B}(\omega, z) \\
& \leq 2 M_{B} \# B_{B}\left(a_{i}, Q_{1}+Q_{2}\right) \sum_{d_{B}\left(a_{i}, \omega\right) \leq Q_{1}+Q_{2}} \nabla^{B} k_{\ell-1}^{B}(\omega, z) .
\end{aligned}
$$

Then we obtain

$$
\begin{aligned}
I_{2}(n, x) \leq & C^{\prime} \sum_{z \in V} e^{8 t_{\nu} d^{2}(x, z) / n} \\
& \sum_{\ell_{1}=1}^{n} \sum_{i=+,-} \sum_{a_{1 i} \in A_{i}} \sup _{d(x, \omega) \leq 1} k_{n-\ell_{1}}\left(\omega, a_{1 i}\right) \sum_{d_{B}\left(a_{1 i}, \omega_{1}\right) \leq Q_{1}+Q_{2}} \nabla^{B} k_{\ell_{1}-1}^{B}\left(\omega_{1}, z\right) \\
& \sum_{\ell_{2}=1}^{n} \sum_{j=+,-} \sum_{a_{2 j} \in A_{j}} \nabla k_{n-\ell_{2}}\left(x, a_{2 j}\right) \sum_{d_{B}\left(a_{2 j}, \omega_{2}\right) \leq Q_{1}+Q_{2}} \nabla^{B} k_{\ell_{2}-1}^{B}\left(\omega_{2}, z\right) m_{B}(z)^{2} m(z)^{-1} .
\end{aligned}
$$




\section{Lemma 2.2}

$$
\begin{aligned}
& \sum_{\ell_{2}=1}^{n} \sum_{j=+,-} \sum_{a_{2 j} \in A_{j}} \nabla k_{n-\ell_{2}}\left(x, a_{2 j}\right) \sum_{d_{B}\left(a_{2 j}, \omega_{2}\right) \leq Q_{1}+Q_{2}} \nabla^{B} k_{\ell_{2}-1}^{B}\left(\omega_{2}, z\right) m_{B}(z)^{2} m(z)^{-1} \\
\leq & \frac{C\left(t_{\nu}\right) T_{\nu}+C}{\sqrt{n} V(x, \sqrt{n})}+\frac{C\left(t_{\nu}\right) T_{\nu}}{\sqrt{n} V(z, \sqrt{n})} .
\end{aligned}
$$

Proof. From the induction hypothesis for $\nabla k_{n}$ and the Gaussian upper bound for $\nabla^{B} k_{n}^{B}$,

$$
\begin{aligned}
& \sum_{\ell_{2}=1}^{n} \sum_{j=+,-} \sum_{a_{2 j} \in A_{j}} \nabla k_{n-\ell_{2}}\left(x, a_{2 j}\right) \sum_{d_{B}\left(a_{2 j}, \omega_{2}\right) \leq Q_{1}+Q_{2}} \nabla^{B} k_{\ell_{2}-1}^{B}\left(\omega_{2}, z\right) m_{B}(z)^{2} m(z)^{-1} \\
\leq & \sum_{i=+,-} \sum_{a_{i} \in A_{i}}\left\{\frac{T_{\nu}}{\sqrt{n-1} V(x, \sqrt{n-1})} e^{-t_{\nu} d^{2}\left(x, a_{i}\right) /(n-1)} \chi_{B_{B}\left(a_{i}, Q_{1}+Q_{2}+1\right)}(z)\right. \\
& \sum_{\ell=2}^{n-1} \frac{T_{\nu}}{\sqrt{n-\ell} V(x, \sqrt{n-\ell})} e^{-t_{\nu} d^{2}\left(x, a_{i}\right) /(n-\ell)} \frac{C}{\sqrt{\ell-1} V_{B}(z, \sqrt{\ell-1})} e^{-c d_{B}^{2}\left(a_{i}, z\right) /(\ell-1)} \\
& \left.+\chi_{B(x, 1)}\left(a_{i}\right) \frac{C}{\sqrt{n-1} V_{B}\left(a_{i}, \sqrt{n-1}\right)} e^{-c d_{B}^{2}\left(a_{i}, z\right) /(n-1)}\right\} \\
\leq & \frac{C^{\prime} T_{\nu}}{\sqrt{n-1} V(x, \sqrt{n-1})} e^{-c d_{B}^{2}\left(a_{i}, z\right) /(\ell-1)} \\
& +\sum_{\ell=2}^{n / 2} \sum_{i=+,-} \sum_{a_{i} \in A_{i}} \frac{C^{\prime} T_{\nu}}{\sqrt{n} V(x, \sqrt{n})} \frac{T^{\prime}}{\sqrt{\ell-1} V_{B}(z, \sqrt{\ell-1})} \\
& +\sum_{\ell \geq n / 2}^{n-1} \sum_{i=+,-} \sum_{a_{i} \in A_{i}} \frac{T_{\nu}}{\sqrt{n-\ell} V(x, \sqrt{n-\ell})} e^{-t_{\nu} d^{2}\left(x, a_{i}\right) /(n-\ell)} \frac{C^{\prime}}{\sqrt{n} V_{B}(z, \sqrt{n})} \\
& +\frac{C^{\prime}}{\sqrt{n-1} V_{B}(x, \sqrt{n-1})} e^{-c^{\prime} d_{B}^{2}(x, z) /(n-1)} .
\end{aligned}
$$

Since

$$
\begin{aligned}
\sum_{a_{i} \in A_{i}} e^{-t_{\nu} d^{2}\left(x, a_{i}\right) / \ell} & =\sum_{k=0}^{\infty} \sum_{k \leq d^{2}\left(x, a_{i}\right) / \ell<k+1} e^{-t_{\nu} d^{2}\left(x, a_{i}\right) / \ell} \\
& \leq \sum_{k=0}^{\infty} \#\left\{a \in A \mid t_{\nu} d^{2}\left(x, a_{i}\right) / \ell \leq k+1\right\} e^{-k} \\
& \leq \sum_{k=0}^{\infty} V_{A}\left(x, \sqrt{t_{\nu}^{-1}(k+1) \ell}\right) e^{-k}
\end{aligned}
$$


and $V_{B}(x, r)$ is comparable with $V(x, r),(14)$ is less than

$$
\begin{aligned}
& \frac{C^{\prime} T_{\nu}+C}{\sqrt{n-1} V(x, \sqrt{n-1})} \\
& +\frac{C^{\prime} T_{\nu}}{\sqrt{n} V(x, \sqrt{n})} \sum_{\ell=2}^{n / 2} \sum_{k=0}^{\infty} \frac{C V_{A}\left(z, \sqrt{c^{-1}(k+1)(\ell-1)}\right)}{\sqrt{\ell-1} V_{B}(z, \sqrt{\ell-1})} e^{-k} \\
& +\sum_{\ell \geq n / 2}^{n-1} \sum_{k=0}^{\infty} \frac{T_{\nu} V_{A}\left(x, \sqrt{t_{\nu}^{-1}(k+1)(n-\ell)}\right)}{\sqrt{n-\ell} V(x, \sqrt{n-\ell})} e^{-k} \frac{C^{\prime}}{\sqrt{n} V_{B}(z, \sqrt{n})} .
\end{aligned}
$$

By the volume doubling property on $X$,

$$
\begin{aligned}
& \sum_{\ell=2}^{n / 2} \sum_{k=0}^{\infty} \frac{V_{A}\left(z, \sqrt{c^{-1}(k+1)(\ell-1)}\right)}{\sqrt{\ell-1} V_{B}(z, \sqrt{\ell-1})} e^{-k} \\
= & \sum_{\ell=2}^{n / 2} \sum_{k=0}^{\infty} \frac{V_{A}\left(z, \sqrt{c^{-1}(k+1)(\ell-1)}\right)}{V\left(z, \sqrt{c^{-1}(k+1)(\ell-1)}\right)} \cdot \frac{V\left(z, \sqrt{c^{-1}(k+1)(\ell-1)}\right)}{\sqrt{\ell-1} V_{B}(z, \sqrt{\ell-1})} e^{-k} \\
\leq & \sum_{k=0}^{\infty}\left(\sum_{\ell=2}^{n / 2} \frac{V_{A}\left(z, \sqrt{c^{-1}(k+1)(\ell-1)}\right)}{\sqrt{\ell-1} V\left(z, \sqrt{c^{-1}(k+1)(\ell-1)}\right)}\right) C\left(c^{-1}(k+1)\right)^{\delta} e^{-k}
\end{aligned}
$$

for some $\delta>0$. From the assumption (7) for $V_{A}$ in Theorem 1, this is uniformly bounded for $z \in V$. Then the lemma follows.

By this lemma and the Gaussian upper bounds for $\nabla^{B} k_{n}^{B}$ and $k_{n}, I_{2}$ is estimated by

$$
\begin{aligned}
& C^{\prime} \sum_{z \in V} e^{8 t_{\nu} d^{2}(x, z) / n}\left\{\sum_{i=+,-a_{1 i} \in A_{i}} \frac{C}{V(x, \sqrt{n-1})} e^{-c d^{2}\left(x, a_{1 i}\right) /(n-1)} \chi_{B_{B}\left(a_{1 i}, Q_{1}+Q_{2}+1\right)}(z)\right. \\
& +\sum_{\ell_{1}=2}^{n-1} \sum_{i=+,-a_{1 i} \in A_{i}} \sum_{V\left(x, \sqrt{n-\ell_{1}}\right)} e^{-c d^{2}\left(x, a_{1 i}\right) /\left(n-\ell_{1}\right)} \\
& \quad \times \frac{C}{\sqrt{\ell_{1}-1} V_{B}\left(a_{1 i}, \sqrt{\ell_{1}-1}\right)} e^{-c d_{B}^{2}\left(a_{1 i}, z\right) /\left(\ell_{1}-1\right)} \\
& \left.+\sum_{i=+,-a_{1 i} \in A_{i}} \chi_{B(x, 1)}\left(a_{1 i}\right) \frac{C}{\sqrt{n-1} V_{B}\left(a_{1 i}, \sqrt{n-1}\right)} e^{-c d_{B}^{2}\left(a_{1 i}, z\right) /(n-1)}\right\} \\
& \times\left(\frac{C\left(t_{\nu}\right) T_{\nu}+C}{\sqrt{n} V(x, \sqrt{n})}+\frac{C\left(t_{\nu}\right) T_{\nu}}{\sqrt{n} V(z, \sqrt{n})}\right) .
\end{aligned}
$$

Since

$$
e^{8 t_{\nu} d^{2}(x, z) / n} \leq e^{16 t_{\nu} d^{2}\left(x, a_{1 i}\right) /\left(n-\ell_{1}\right)} e^{32 Q_{1}^{2} Q_{2}^{2} t_{\nu}} e^{32 Q_{1}^{2} t_{\nu} d_{B}^{2}\left(a_{1 i}, z\right) /\left(\ell_{1}-1\right)}
$$


for $1<\ell_{1}<n$,

$$
\begin{aligned}
& I_{2}(n, x) \leq C^{\prime \prime} \sum_{z \in V}\left\{\sum_{i=+,-a_{1 i} \in A_{i}} \sum_{V(x, \sqrt{n-1})} e^{-\left(c-16 t_{\nu}\right) d^{2}\left(x, a_{1 i}\right) /(n-1)} \chi_{B_{B}\left(a_{1 i}, Q_{1}+Q_{2}+1\right)}(z)\right. \\
& +\sum_{\ell_{1}=2}^{n-1} \sum_{i=+,-} \sum_{a_{1 i} \in A_{i}} \frac{C}{V\left(x, \sqrt{n-\ell_{1}}\right)} e^{-\left(c-16 t_{\nu}\right) d^{2}\left(x, a_{1 i}\right) /\left(n-\ell_{1}\right)} \\
& \times \frac{C}{\sqrt{\ell_{1}-1} V_{B}\left(a_{1 i}, \sqrt{\ell_{1}-1}\right)} e^{-\left(c-32 Q_{1}^{2} t_{\nu}\right) d_{B}^{2}\left(a_{1 i}, z\right) /\left(\ell_{1}-1\right)} \\
& \left.+\sum_{i=+,-} \sum_{a_{1 i} \in A_{i}} \chi_{B(x, 1)}\left(a_{1 i}\right) \frac{C}{\sqrt{n-1} V_{B}\left(a_{1 i}, \sqrt{n-1}\right)} e^{-\left(c-32 Q_{1}^{2} t_{\nu}\right) d_{B}^{2}\left(a_{1 i}, z\right) /(n-1)}\right\} \\
& \times\left(\frac{C\left(t_{\nu}\right) T_{\nu}+C}{\sqrt{n} V(x, \sqrt{n})}+\frac{C\left(t_{\nu}\right) T_{\nu}}{\sqrt{n} V(z, \sqrt{n})}\right) .
\end{aligned}
$$

By the volume doubling property for $V$,

$$
\begin{aligned}
\frac{1}{V(z, \sqrt{n})} e^{-c d^{2}(a, z) / n} & =\frac{1}{V(a, \sqrt{n})} \cdot \frac{V(a, \sqrt{n})}{V(z, \sqrt{n})} e^{-c d^{2}(a, z) / n} \\
& \leq \frac{1}{V(a, \sqrt{n})} \cdot \frac{V(z, \sqrt{n}+d(a, z))}{V(z, \sqrt{n})} e^{-c d^{2}(a, z) / n} \\
& \leq \frac{1}{V(a, \sqrt{n})}\left(1+\frac{d(a, z)}{\sqrt{n}}\right)^{\delta} e^{-c d(a, z) / n} \\
& \leq \frac{C}{V(a, \sqrt{n})} e^{-c d^{2}(a, z) / 2 n}
\end{aligned}
$$

For sufficiently small $t_{\nu}>0, I_{2}$ is estimated by

$$
\begin{aligned}
& \left\{\sum_{i=+,-a_{1 i} \in A_{i}} \sum_{V(x, \sqrt{n-1})} e^{-\left(c-16 t_{\nu}\right) d^{2}\left(x, a_{1 i}\right) /(n-1)}\right. \\
& +C \sum_{\ell_{1}=2}^{n / 2} \sum_{i=+,-a_{1 i} \in A_{i}} \sum_{V(x, \sqrt{n})} e^{-\left(c-16 t_{\nu}\right) d^{2}\left(x, a_{1 i}\right) / n} \frac{C}{\sqrt{\ell_{1}-1}} \\
& +C \sum_{\ell_{1}>n / 2}^{n-1} \sum_{i=+,-a_{1 i} \in A_{i}} \sum_{V\left(x, \sqrt{n-\ell_{1}}\right)} e^{-\left(c-16 t_{\nu}\right) d^{2}\left(x, a_{1 i}\right) /\left(n-\ell_{1}\right)} \frac{C^{\prime}}{\sqrt{n}} \\
& \left.+\frac{C}{\sqrt{n-1}}\right\} \frac{C\left(t_{\nu}\right) T_{\nu}+C\left(t_{\nu}\right)}{\sqrt{n} V(x, \sqrt{n})} .
\end{aligned}
$$

Here we remark that

$$
\sum_{z \in V} \frac{1}{V(a, \sqrt{\ell})} e^{-c d^{2}(a, z) / \ell} \leq C \quad \forall \ell \geq 1 .
$$


By the same argument in Lemma 2.2 with the assumption (7) of the Theorem, we conclude that

$$
\begin{aligned}
I_{2}(n, x) \leq & C\left\{\sum_{k=0}^{\infty} \frac{\sqrt{c\left(t_{\nu}\right)(k+1)(n-1)} V_{A}\left(x, \sqrt{c\left(t_{\nu}\right)(k+1)(n-1)}\right)}{V\left(x, \sqrt{c\left(t_{\nu}\right)(k+1)(n-1)}\right)}\left(c\left(t_{\nu}\right)(k+1)\right)^{\delta} e^{-k}\right. \\
& +\sum_{k=0}^{\infty} \frac{c\left(t_{\nu}\right)(k+1)(n-1) V_{A}\left(x, \sqrt{c\left(t_{\nu}\right)(k+1)(n-1)}\right)}{V\left(x, \sqrt{c\left(t_{\nu}\right)(k+1)(n-1)}\right)}\left(c\left(t_{\nu}\right)(k+1)\right)^{\delta} e^{-k} \\
& \left.+\sum_{k=0}^{\infty} \sum_{\ell_{1}>n / 2}^{n-1} \frac{V_{A}\left(x, \sqrt{c\left(t_{\nu}\right)(k+1)\left(n-\ell_{1}\right)}\right)}{V\left(x, \sqrt{c\left(t_{\nu}\right)(k+1)\left(n-\ell_{1}\right)}\right)}\left(c\left(t_{\nu}\right)(k+1)\right)^{\delta} e^{-k}+\frac{\sqrt{n}}{\sqrt{n-1}}\right\} \\
& \cdot \frac{C\left(t_{\nu}\right) T_{\nu}+C^{\prime}\left(t_{\nu}\right)}{n V(x, \sqrt{n})} \\
\leq & M \frac{C^{\prime}\left(t_{\nu}\right) T_{\nu}+C^{\prime \prime}\left(t_{\nu}\right)}{n V(x, \sqrt{n})}
\end{aligned}
$$

which gives a desired bound for (11).

\subsection{Conclusion of induction}

From the above arguments, for small $t_{\nu}>0$, we obtain

$$
\nabla k_{\nu}(x, y) \leq \frac{\left(C\left(t_{\nu}\right) T_{\nu}+C^{\prime}\left(t_{\nu}\right)\right)^{1 / 2}}{\sqrt{\nu} V(x, \sqrt{\nu})^{1 / 2}} \frac{C\left(t_{\nu}\right)}{V(y, \sqrt{\nu})^{1 / 2}} e^{-2 t_{\nu} d(x, y)^{2} / \nu}
$$

under the hypothesis that

$$
\nabla k_{i}(x, y) \leq \frac{T_{\nu}}{\sqrt{i} V(x, \sqrt{i})} e^{-t_{\nu} d(x, y)^{2} / i}
$$

for $1 \leq i \leq \nu-1$. Since $X$ has the volume doubling property, by the same arguments as in the proof of Theorem 5.2 in [3], we have

$$
\nabla k_{\nu}(x, y) \leq \frac{C^{\prime \prime}\left(t_{\nu}\right)\left(C\left(t_{\nu}\right) T_{\nu}+C^{\prime}\left(t_{\nu}\right)\right)^{1 / 2}}{\sqrt{\nu} V(x, \sqrt{\nu})} e^{-t_{\nu} d(x, y)^{2} / \nu} .
$$

Finally, by taking $T_{\nu}$ large enough so that

$$
C^{\prime \prime}\left(t_{\nu}\right)\left(C\left(t_{\nu}\right) T_{\nu}+C^{\prime}\left(t_{\nu}\right)\right)^{1 / 2} \leq T_{\nu},
$$

we obtain

$$
\nabla k_{\nu}(x, y) \leq \frac{T_{\nu}}{\sqrt{\nu} V(x, \sqrt{\nu})} e^{-t_{\nu} d(x, y)^{2} / \nu} .
$$

Then the proof of theorem is complete. 


\section{Acknowledgment}

This work was done while the author stayed in the University of Cergy-Pontoise by the support of the Overseas Advanced Educational Research Practice Support Program by Japanese Ministry of Education, Culture, Sports, Science and Technology. He would like to express his gratitude to Professor Thierry Coulhon for useful comments, encouragements and a lot of support during his stay.

\section{References}

[1] Auscher, P., Coulhon, T., Duong, X. T., Hofmann, S.: 'Riesz transform on manifolds and heat kernel regularity', Ann. Sci. École Norm. Sup. (4) 37 (2004), no. 6, 911-957.

[2] Coulhon, T., Duong, X. T.: 'Riesz transforms for $1 \leq p \leq 2$ ', Trans. Amer. Math. Soc. 351 (1999), no. 3, 1151-1169.

[3] Coulhon, T., Grigor'yan, A.: 'Random walks on graphs with regular volume growth', GAFA $\underline{8}$ (1998), 656-701.

[4] Coulhon, T., Dungey, N.: 'Riesz transforms on perturbations', preprint.

[5] Dungey, N.: 'Heat kernel estimates and Riesz transforms on some Riemannian covering manifolds', Math. Z. 247 (2004), no. 4, 765-794.

[6] Dungey, N.: 'Some gradient estimates on covering manifolds', Bull. Pol. Acad. Sci. Math. 52 (2004), no. 4, 437-443.

[7] Hebisch, W., Saloff-Coste, L.: 'Gaussian estimates for Markov chains and random walks on groups', Ann. Probab. 21 (1993), no. 2, 673-709.

[8] Ishiwata, S.: 'A Berry-Esseen type theorem on nilpotent covering graphs', Canad. J. Math. $\underline{56}$ (2004), no. 5, 963-982.

[9] Ishiwata, S.: 'Discrete version of Dungey's proof for the gradient heat kernel estimate on coverings', Annales Mathématiques Blaise Pascal. 14 (2007), no. $1,93-102$.

[10] Ishiwata, S.: 'Connectivity on discrete nilpotent groups', in preparation.

[11] Kanai, M.: 'Rough isometries, and combinatorial approximations of geometries of non-compact riemannian manifolds', J. Math. Soc. Japan 37 (1985), 391-413.

[12] Li, H. Q.: 'La transformation de Riesz sur les variétés coniques. J. Funct. Anal. 168 (1999), no. 1, 145-238.

[13] Russ, E.: 'Riesz transforms on Graphs for $1 \leq p \leq 2$ ', Math. Scand. $\underline{87}$ (2000), 133-160.

[14] Russ, E.: 'Riesz transforms on graphs for $p \geq 2$ ', preprint. 